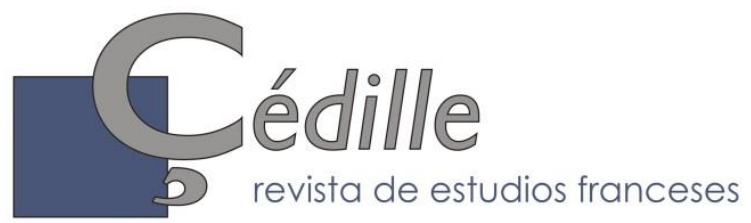

ISSN: 1699-4949

$n^{0} 18$ (otoño de 2020)

Monografías 11

Epistemocrítica: análisis literario y saber científico Amelia Gamoneda Lanza \& Francisco González Fernández, editores científicos

\title{
El epistemotexto literario
}

\author{
Jesús CAMARERo \\ Universidad del País Vasco \\ jesus.camarero@ehu.eus \\ ORCID: 0000-0003-2714-7718
}

\section{Resumen}

Desde el punto de vista de la Epistemocrítica, el texto literario es considerado como una encrucijada donde se encuentran diferentes saberes en una dinámica transversal (transconocimiento). Esta definición remite al concepto de «epistemotexto», una categoría de la metodología de análisis epistemocrítico, y se extiende al ámbito de la ficcionalidad literaria (epistemoficción), considerada aquí un sistema de conocimiento desde el punto de vista epistemológico.

Palabras clave: Epistemocrítica, Narratividad, Ficcionalidad, Jules Verne, Gustave Flaubert, Georges Perec.

\section{Résumé}

Du point de vue de l'Épistémocritique, le texte littéraire est considéré comme un carrefour où se retrouvent des savoirs différents à dynamique transversale (transconnaissance). Cette définition renvoie donc au concept d' " épistémotexte », une catégorie de la méthodologie d'analyse épistémocritique, et elle est rapportée au domaine de la fictionnalité littéraire (épistémofiction), considérée ici comme un système de connaissance du point de vue épistémologique.

Mots-clé: Épistémocritique, Narrativité, Fictionnalité, Jules Verne, Gustave Flaubert, Georges Perec.

\begin{abstract}
From the point of view of Epistemocriticism, a literary text is considered to be at a crossroads where different types of knowledge combine in a transverse dynamics (transknowledge). Such definition refers to the concept of «epistemotext», and extends to the field of literary fictionality (epistemofiction), a knowledge-based system from an epistemological perspective.
\end{abstract}

Key words: Epistemocriticism, Narrativity, Fictionality, Jules Verne, Gustave Flaubert, Georges Perec.

\footnotetext{
* Artículo recibido el 25/02/2020, aceptado el 25/10/2020.
} 


\section{Introducción}

A día de hoy, la epistemocrítica, como metodología crítica literaria, ha recorrido ya un camino suficientemente largo desde sus comienzos, allá por 1990, cuando Michel Pierssens publicó un ensayo titulado Savoirs à l'œuvre. Essais d'épistémocritique. Sus investigaciones sobre la relación entre la literatura y la ciencia abarcan aportaciones que tienen que ver con la relación entre la literatura y la neurociencia, las ciencias humanas y las ciencias naturales y experimentales. En este sentido, el ensayo de Jonah Lehrer, Proust y la neurociencia, puede servir perfectamente de ejemplo para demostrar que las líneas de investigación epistemocríticas son de alto interés, pues la obra de Lehrer constituye una investigación sobre varios artistas -Walt Whitman, George Eliot, Auguste Escoffier, Marcel Proust, Paul Cézanne, Igor Stravinski, Gertrude Stein, Virginia Woolf- que, como el mismo autor señala, «se adelantaron a los descubrimientos de la neurociencia» (Lehrer, 2010: 15).

De este modo, la epistemocrítica, enmarcada en el ámbito general del conocimiento, podría haber constituido, por ejemplo, una respuesta eficaz a la duda de Thomas S. Kuhn en el inicio mismo de sus tareas investigadoras y que le llevó a publicar su obra La estructura de las revoluciones científicas en relación con los múltiples ámbitos del conocimiento: «Tanto la historia como mis conocimientos me hicieron dudar de que quienes practicaban las ciencias naturales poseyeran respuestas más firmes o permanentes para esas preguntas que sus colegas en las ciencias sociales» (Kuhn, 1995: 13).

Esto viene a colación también porque, precisamente en esa misma línea de sus teorías sobre la ciencia, y sobre todo de su teoría del «paradigma», sería posible plantear la hipótesis de un «doble paradigma» para la epistemocrítica. El comentario de Kuhn confirma efectivamente la relación ya descrita entre literatura y ciencia, pero también abre la posibilidad de pensar la literatura como una dimensión de amplio espectro dentro de la relación literatura-ciencia, en la que la literatura bien podría constituir también un dominio epistémico autónomo.

Así que, según ese doble paradigma de la epistemocrítica, una vía sería la ya descrita más arriba sobre la relación definible como «la literatura "y" la ciencia». Otra vía, directamente relacionada con la anterior, sería definible como «la literatura "como" ciencia»: puesto que la literatura no solo no es ajena, sino que está absolutamente implicada en un juego epistemológico con la ciencia (como así lo demuestra la primera vía), se podría contemplar la posibilidad de que la literatura pudiera funcionar -también- como ciencia por sí misma y en sí misma (incluso como presciencia o metaciencia), de modo que pudiera ser considerada una modalidad científica que se ocuparía lógicamente de la construcción del saber y del conocimiento. Desde el punto de vista metodológico, quizá no lo haría siguiendo exactamente el mismo procedimiento de las otras ciencias (impulso heurístico), aunque sin duda podría fácilmente adaptar los principios de funcionamiento de los otros saberes y utilizar a la vez sus propias herramientas.

Por lo tanto, sin dejar de lado la primera vía del paradigma epistemocrítico, este trabajo se propone la exploración de la segunda vía, su hipótesis y su po- 
sible desarrollo. La factibilidad del proyecto se entrevé por ejemplo en una obra de Aldous Huxley de 1963, cuando en un ensayo seminal sobre la literatura y la ciencia destaca la aportación de la literatura en relación con el saber científico. Por un lado, la ciencia examina «una serie de casos particulares, anota todas las similitudes y uniformidades y abstrae de estas una generalización» (Huxley, 2017: 13); por otro lado, a la literatura le conciernen «la descripción de las apariencias y las cualidades observables de los objetos percibidos como totalidades, los juicios, las comparaciones y discriminaciones, la naturaleza interior y las esencias, y, finalmente $[\ldots]$ el no pensamiento, la atemporal mismidad (o unicidad del ser)» (Huxley, 2017: 15).

Por su parte, Jonah Lehrer reconoce la presciencia de la literatura respecto a ciertos avances científicos y, refiriéndose a Proust, señala que «sólo el artista estaba capacitado para describir la realidad tal y como era de hecho experimentada» (Lehrer, 2010: 105). No obstante, tampoco duda en señalar una dinámica diferenciada de ambos dominios respecto del conocimiento: «Los artistas urden bonitos relatos, mientras que los científicos describen objetivamente el universo» (Lehrer, 2010: 16). En cualquier caso, queda abierta la posibilidad del doble dominio epistémico de la literatura, bien en interacción con la ciencia, bien de un modo autónomo.

\section{Literatura «y» ciencia}

\subsection{Literariedad y epistemocrítica: la epistemotextualidad}

$\mathrm{Ni}$ que decir tiene que en el ámbito epistemocrítico destaca la obra de un autor, Jules Verne, cuyos relatos constituyen el paradigma de una narrativa arraigada en todo tipo de saberes científicos que el autor ilustró de modo extraordinario en la mayoría de sus textos. Verne representa al hombre ilustrado de su época. De este modo, como sigue muy de cerca los descubrimientos e invenciones que se producen sin cesar, la información recopilada pasa a conformar la base de sus relatos, en general por medio de extrapolaciones que a veces son coincidentes con la realidad futura, aunque no siempre se trate de una auténtica pre-visión. En una novela como Les cinq cents millions de la Bégum aparece, por ejemplo, el prototipo del primer satélite artificial, aunque en realidad se trata de un obús: Verne no llega a exponer realmente la tecnología de los nuevos inventos, sino que solo los evoca y de paso los explota hábilmente en modo narrativo para mostrar su poder o su efecto tanto en el nivel de la historia como en su interpretación más allá incluso de la misma.

Conviene pues distinguir entre los cambios - sin duda revolucionarios - de la ciencia en el siglo XIX, y su pre-visión en relación con el futuro (presciencia), sobre todo con el siglo XX. Desde este punto de vista, Verne no construye relatos científicos stricto sensu. Ahora bien, pergeña unas historias en las que queda maravillosamente mitificada la modernidad científica y tecnológica de su época:

Modestes pionniers de la science, nous étions heureux dans notre obscurité. Le serons-nous encore ? Non, peut-être, à moins... Mais je n'ose te parler d'une idée arrêtée dans ma pensée... a moins que cette fortune même ne devienne en 
nos mains un nouvel et puissant appareil scientifique, un outil prodigieux de civilisation! (Verne, 2002: 20).

Eso sí, debido al innegable enfoque humanista de la obra verniana, queda en evidencia también el conflicto surgido entre el hombre y las máquinas, que ciertamente se va a convertir en un problema de graves dimensiones en el siglo XXI. Por eso, la obra de Verne puede ser considerada una aportación capital a la teoría general del humanismo, porque incide en el análisis y evidenciación de algunos problemas que vienen afectando al ser humano desde casi el mismo comienzo de nuestra modernidad.

Según distintos principios de la literariedad relacionados directamente con el conocimiento (heuresis, mundos posibles, autognosis, representación, mimesis), todo lo descrito más arriba es llevado a cabo por la literatura mediante relatos, historias narradas, ficciones o fabulaciones. Eso sí, desde el punto de vista hermenéutico, la importancia de dichos relatos estriba sobre todo en la adecuada interpretación de su contenido, que además puede ser entendido de modo diferente según el contexto y la época. Puesto que la transmisión y hasta la producción del conocimiento se puede efectuar por medio de la literatura y -como luego se veráen su mismo proceso de textualización y recepción, la función epistémica puede ser propia de la literatura y formar parte entonces de la literariedad. Es decir que el hecho de que un texto literario pueda tener una función en relación directa con el saber (transconocimiento), implica que esa misma función es uno de los rasgos determinantes de la literariedad.

Efectivamente, desde una perspectiva más específica o especializada, la literatura recoge, transporta y trascendentaliza en sus textos multitud de saberes científicos de todas las épocas («epistemotextos»). Esto es así porque los autores son siempre grandes lectores. En algunos casos, son también conocedores o especialistas en determinados temas, pero en otros simplemente trasmiten grandes y valiosas intuiciones derivadas de la experiencia y el saber acumulados por un autor a lo largo de una vida entera dedicada a profundizar en las grandes verdades del ser humano y del mundo, si no de otras dimensiones en principio desconocidas para la mayoría de la humanidad. Este ámbito de la práctica y de la recepción literaria específica en cuanto al saber científico en su relación con el texto se podría denominar «epistemotextualidad».

Así pues, por ejemplo, el epistemotexto de Verne en Les cinq cents millions de la Bégum consiste en la referenciación por medio del relato de múltiples saberes, todos ellos debidamente contextualizados en el argumento de la obra, de la que forman parte narrativamente, ya que articulan el sentido de la misma en cuanto a las estructuras del contenido y más precisamente en cuanto a sus líneas temáticas. Pero, además, desde el punto de vista estrictamente epistemocrítico, suponen la aportación de un transconocimiento de alto valor. En este apartado, destaca el saber relativo a la medicina: el protagonista, el doctor Sarrasin, es un médico, y además humanista. Sobre todo se hace referencia a un tema que resulta de la interacción medicina-humanismo (el racismo), pero también hay múltiples referencias a la higiene, un tema capital en la época que, por otra parte, entronca 
perfectamente con el argumento inicial que sostiene toda la novela, la construcción de la ciudad ideal:

Messieurs, parmi les causes de maladie, de misère et de mort qui nous entourent, il faut en compter une à laquelle je crois rationnel d'attacher une grande importance: ce sont les conditions hygiéniques déplorables dans lesquelles la plupart des hommes sont placés. Ils s'entassent dans des villes, dans des demeures souvent privées d'air et de lumière, ces deux agents indispensables de la vie. Ces agglomérations humaines deviennent parfois de véritables foyers d'infection. Ceux qui n'y trouvent pas la mort sont au moins atteints dans leur santé; leur force productive diminue, et la société perd ainsi de grandes sommes de travail qui pourraient être appliquées aux plus précieux usages. [...] Pourquoi ne réunirions-nous pas toutes les forces de notre imagination pour tracer le plan d'une cité modèle sur des données rigoureusement scientifiques ? (Verne, 2002: 41).

Como es lógico, debido al tema de Les cinq cents millions de la Bégum y en general de la mayoría de los relatos vernianos, el saber relacionado con la ingeniería y la tecnología ocupa también un lugar destacado: problemas matemáticos, construcción de armamento pesado, cuestiones avanzadas de balística, estudios de química sobre sustancias explosivas, diseño y funcionamiento de la industria siderúrgica y metalúrgica, telefonía, etc. Además, el relato comienza con sorprendentes alusiones a los sistemas de información de la época, en concreto a la prensa escrita, y en ese contexto aparecen referencias a la modélica prensa inglesa e incluso a las fake news. Sin olvidar por supuesto las referencias a la teoría política: ideología racista, democracia ciudadana, gobernanza y teoría del humanismo. El aparato epistémico se completa, entre otras, con aportaciones diversas de la psicología, la botánica, la hipología y la economía.

A lo largo ya de siglos, la creación literaria ha venido aportando textos literarios -epistemotextos- que, sin perjuicio de su demostrada función epistémica, responden a variantes más bien de tipo pragmático. Aunque a continuación se citen solo algunos ejemplos de esta variedad subgenérica, que viene a enriquecer la definición de la epistemotextualidad, los epistemotextos literarios ofrecen un amplio abanico de posibilidades en sus estrategias o formas de articular contenido epistémico.

En primer lugar, una serie de epistemotextos en los que la literatura está en relación con saberes ya existentes, admitidos o «legitimados», en expresión de Jean-François Lyotard (1984: 22-23):

a) Fruto de la vinculación directa que en origen mantenía la literatura con la filosofía, e incluso con la ciencia, en los primeros tiempos de su historia, los Essais de Michel de Montaigne constituyen un buen ejemplo de epistemotexto literario-filosófico, que coincide con la literatura clásica greco-latina.

b) Algunos de los epistemotextos más conocidos o famosos consisten en la descripción de saberes y técnicas de una época determinada, como así lo demues- 
tra la novela Bouvard et Pécuchet de Gustave Flaubert, en este caso en relación sobre todo con la agronomía.

c) Hay epistemotextos cuya narración está basada en saberes especializados, como es el caso de la neurociencia de principios del siglo XX en $\grave{A}$ la recherche du temps perdu de Marcel Proust.

d) Algunos epistemotextos asumen como función epistémica propia la crítica de saberes que son considerados, en ese momento, obsoletos o disfuncionales, como en Le malade imaginaire de Molière, una crítica feroz de la medicina del siglo XVII.

Y en segundo lugar, epistemotextos en los que la narración literaria está al servicio de una propuesta de conocimiento, una búsqueda o anticipación del saber futuro, por mucho que no siempre esta visión sea positiva o siquiera posible:

e) Aquellos epistemotextos que se conforman como relatos que representan saberes todavía inexistentes o no culminados, planteados entonces como presciencia, cual es el caso de Les cinq cents millions de la Bégum de Jules Verne.

f) Los epistemotextos que responden al género literario de la cienciaficción y de la utopía-distopía y que describen saberes «a futuro», como es el caso de la tecnópolis en 1984 de George Orwell.

Toda la tipología epistemotextual descrita más arriba responde efectivamente a unos textos literarios en los que el nivel de las estructuras del contenido adquiere una importancia principal, incluso sobrepasando el nivel de la forma, que en literatura ayuda a definir bien la literariedad de cualquier texto. Ernst Robert Curtius, en su magna obra Literatura europea y Edad Media latina, ya se refería a los «manierismos de contenido», que consideraba hermanos de los manierismos formales, en los que destaca la literatura española del Siglo de Oro. Para Curtius (1981: 410-411) esta dimensión del contenido adquiría su importancia como idea ingeniosa o sorprendente (el epigrama), pero la subrayaba también por su «agudeza», o sea por su capacidad para generar ideas nuevas, fruto de la genialidad residida en lo que se cuenta, un contenido sapiencial.

El epistemotexto atiende, pues, a esas estructuras del contenido del texto literario, y lo hace en relación con otros saberes, en el ámbito del conocimiento. Este trans-conocimiento es lo que representa o contiene el epistemo-texto en su discurso, por mucho que este corresponda al ámbito de la ficción. De hecho, se puede conceptualizar el epistemotexto como un fenómeno (Camarero, 2015: 3) de doble efecto: en primer lugar, es el productor, el depositario y el transmisor de los saberes en él contenidos; y en segundo lugar, está implicado en su propia recepción por el lector, que no solo infiere esos saberes, sino que pone su propio saber en interacción con lo adquirido para construir una síntesis epistémica en su lectura. Esta síntesis se genera en dos fases sucesivas y complementarias: una primera fase, de transferencia epistémica, en la que el autor deposita en su obra un determinado saber que, mediante la escritura, permanecerá estabilizado y protegido a lo largo del tiempo (grafein); y una segunda fase, de capilaridad cognitiva, en la que el lector, mediante la lectura, se apropia el saber antes depositado en la obra, inte- 
grando en esa misma maniobra su propio saber. El epistemotexto sería el constructo fenoménico (hipermetatexto) producido finalmente por esa síntesis.

Alexandre Koyré, en su estudio Du monde clos à l'univers infini, se refiere a una fase histórica, los siglos XVI y sobre todo XVII, cuando la filosofía y la ciencia avanzan juntas, hasta el punto de que separadas «elles deviennent incompréhensibles» (Koyré, 1962: 9). Pero, tras ese momento, los cambios que se producen son de tal calibre que

l'homme, ainsi qu'on le dit parfois, a perdu sa place dans le monde ou, plus exactement peut-être, a perdu le monde même qui formait le cadre de son existence et l'objet de son savoir, et a dû transformer et remplacer non seulement ses conceptions fondamentales mais jusqu'aux structures mêmes de sa pensée» (Koyré, 1962: 11, el resaltado es mío).

$\mathrm{Ni}$ que decir tiene que la literatura de aquella época y sobre todo la posterior debió acusar semejante transformación del paradigma, lo cual demuestra la inevitable relación epistémica que la literatura se ve obligada a mantener con el conocimiento filosófico y científico (epistemocrítica), pues de lo contrario su discurso (sus relatos, por ejemplo) no podrían ser entendidos ni asumidos o serían incoherentes respecto del saber operativo de los lectores en cada época.

La dimensión epistemocrítica de un texto literario afecta a lo que aquí se denomina trans-conocimiento, es decir la representación del saber o del conocimiento en una obra literaria. Dicho esto, el modo como se efectúa esa representación -en principio artística y semiótica, en relación con el conocimiento y la participación del lector, de modo que la síntesis epistémica aboca a la generación del epistemotexto- implica dos maniobras. Por un lado, si la transversalidad del conocimiento y de todos los saberes hace posible que la literatura pueda constituirse en campo o dominio de interacción en el que distintos saberes se entrecruzan (transconocimiento, «convergencia» según Lehrer, 2010: 15), ello demuestra la inoperatividad de la compartimentación del conocimiento. Y, por otro, la transferencia supone que el conocimiento se incorpora al texto literario (escritura, obradepósito) y, al mismo tiempo, entra en fusión con el conocimiento del lector. De esta doble manera -dentro de la literatura y atravesando el tiempo y el espacio-, entra en relación el humanismo del autor (saber depositado en la obra para su transferencia) con el humanismo del lector (suma total de la transversalidad del saber).

Las relaciones entre los saberes diferentes que interactúan entre sí en el epistemotexto literario y las relaciones entre el conocimiento aportado en una obra y el conocimiento construido por la misma literatura, vienen a mostrar una dimensión de la epistemocrítica que podría relacionarla con la literatura comparada y la intertextualidad. Estos son ámbitos de la ciencia literaria en los que se producen numerosas relaciones de tipo literario, y en los que se expande el ejercicio relacional para alcanzar también otras dimensiones del conocimiento, incluido todo tipo de saberes. La epistemocrítica viene a unirse a las perspectivas que ya concernían de facto a los contenidos trasladados por la obra literaria. Sirva para ilus- 
trar esta dimensión de la epistemocrítica la definición de Henry H.H. Remak en respuesta al problema planteado por René Wellek sobre la crisis de la literatura comparada:

La literatura comparada es el estudio de la literatura más allá de las fronteras de un país particular y el estudio de las relaciones entre la literatura y otras áreas de conocimiento o de opinión, como las artes (i.e., pintura, escultura, arquitectura, música), la filosofía, la historia, las ciencias sociales (i.e., política, economía, sociología), las ciencias naturales, la religión, etc. En resumen, es la comparación de una literatura con otra u otras y la comparación de la literatura con otros ámbitos de la expresión humana» (Remak, 1998: 89, el resaltado es mío).

Ciertamente, desde esta perspectiva, se superan las limitaciones ya típicas de la compartimentación literaria (nacional, genérica, histórica, discursiva, etc.) y así se contempla la literatura como una totalidad mediante el juego relacional y la copresencia textual (reescritura), que son el enfoque propio de la literatura comparada (Weltliteratur) y la intertextualidad (littérature au second degré, transtextualité) respectivamente. Pero, basándose en el potencial relacional del comparatismo y del intertexto, el juego del enfoque epistemocrítico hace aparecer nítidamente la literatura convertida en una encrucijada de saberes (interacción de una pluralidad de discursos sapienciales) y un crisol de conocimiento (saber propio, metaciencia).

A esta idea de totalidad impulsada por la propia literatura se llega también por la vía de la fenomenología y la epistemología, siempre que se tenga en cuenta, holísticamente, el fenómeno de la experiencia humana como un acto total: «La relevancia antropológica de las ficciones se debe a que la vida como tal no forma un todo. Al menos, su sentido depende de una totalidad que es inaccesible a nuestra experiencia ordinaria o científica del tiempo» (Innerarity, 1995: 166). De ahí entonces la necesidad de la literatura como ámbito de conocimiento. A esta conclusión también se llega porque no podemos olvidar que actualmente nos hallamos en una situación de crisis de valores y de conocimiento, en la que «la modernización impulsada por las ciencias experimentales y sus aplicaciones técnicas crea unas carencias en el mundo de la vida -sentido, identidad, orientación- a cuya compensación colaboran las ciencias del espíritu» (Innerarity, 1995: 167).

\subsection{El epistemotexto paradójico}

Puesto que se ha definido el epistemotexto como una categoría «ficcional» y literaria del conocimiento, a modo de contrapunto parecería conveniente prestar atención a un autor y a una obra que son considerados paradigmáticos respecto a la epistemotextualidad, aunque se trate del caso muy particular de un epistemotexto sometido a una paradoja deslumbrante, abrumadora. Así pues, desde un enfoque estrictamente epistemocrítico, se puede afirmar que la obra de Bouvard et Pécuchet de Gustave Flaubert adopta la idea del conocimiento como argumento de su relato o tiene como fundamento de su narratividad el saber, en concreto 
múltiples saberes, en relación sobre todo con la agronomía a finales del siglo XIX. Aunque, justo es reconocerlo, la recepción lectora de la obra y su interpretación se resuelve al fin y al cabo de un modo paradójico en cuanto al saber, pues este aparece como algo inaprensible o inalcanzable, e incluso como una interrogación -no poco sarcástica- sobre la utilidad y el futuro del mismo saber.

Todas las acciones, actividades, experiencias, prácticas, tecnologías, proyectos y experimentos narrados en la novela podrían resumirse mediante esta expresión: «Et, ayant plus d'idées, ils eurent plus de souffrances» (Flaubert, 1966: 39). Se trata entonces de un caso paradójico relativo a un epistemotexto muy conocido, en el que el amplio despliegue de saberes entra en colisión precisamente con el relato de la imposibilidad de obtener el conocimiento. Se podría decir que Bouvard et Pécuchet consiste más bien en una taxonomía (una lista, siempre previa al saber) para una guía presunta de la vida en el campo, porque la enumeración y descripción de tantos saberes y técnicas no se resuelve en nada, no se aprehende todo aquello que se pretende, no culmina en saber real lo que solo era una previsión del conocimiento, bastante lejana al mismo por cierto. Queda, pues, claro que el afán taxonómico no coincide con el objetivo epistémico, porque la lista (pre-visión del sistema del saber) no equivale, en este caso, a la realización misma de los proyectos (objetivo final del saber).

En realidad, todo este juego con el saber trae consigo una gran incógnita que el libro propone y deja pendiente al borde del mismo final del siglo XIX, ya en la fase de la post-modernidad, bajo la forma de una aporía cuyos síntomas no pueden ser pasados por alto. Como bien señala Jean-François Lyotard, en general, el libro de Flaubert también estaría afectado por «el estado de la cultura después de las transformaciones que han afectado a las reglas de juego de la ciencia, de la literatura y de las artes a partir del siglo XIX» (Lyotard, 1984: 9). Pero al mismo tiempo, más concretamente, se trata de una crisis de la noción de relato (científico) y por consiguiente -dada la relación osmótica en este caso de Flaubert entre relato (literario) y conocimiento- del conocimiento mismo, que ya no estaría «legitimado», en expresión de Lyotard, por la incidencia de las transformaciones tecnológicas sobre el saber. De este modo, la naturaleza del saber no queda intacta y la adquisición de este «es indisociable de la formación del espíritu, e incluso de la persona» (Lyotard, 1984: 14-16).

La aporía, obviamente, no se resuelve, pero tiene alguna extensión de interés, también de un modo paradójico, y quizá sorprendente, porque si el saber científico ya no encuentra su legitimación en aquella fecha de los inicios de la postmodernidad, sin embargo el relato de Flaubert sí constituye, en cierto modo, un saber (literario), aunque solo sea para mostrar la crisis deslegitimadora del otro saber (científico). Por lo tanto, de lo dicho se podría deducir que el transconocimiento narrativo de este epistemotexto podría estar «legitimado» de algún modo en el marco de los grandes relatos, ya que «en principio, el saber científico no es todo el saber, siempre ha estado en excedencia, en competencia, en conflicto con otro tipo de saber, que para simplificar llamaremos narrativo» (Lyotard, 1984: 
22). Sobre este tipo de saber se presentará luego la hipótesis del segundo paradigma epistemocrítico.

Pero ya en el siglo IV a.C., por boca de Sócrates, se planteaba en el diálogo Menón (80e) de Platón un problema parecido en los siguientes términos:

¿Te das cuenta del argumento erístico que empiezas a entretejer: que no le es posible a nadie buscar ni lo que sabe ni lo que no sabe? Pues ni podría buscar lo que sabe -puesto que ya lo sabe, y no hay necesidad alguna entonces de búsque$\mathrm{da}-$, ni tampoco lo que no sabe -puesto que, en tal caso, ni sabe lo que ha de buscar (Platón, 2000: 296-297).

Así pues, desde el punto de vista epistemológico, esta aporía platónica relativa a la imposibilidad del conocimiento permitiría interpretar stricto sensu la obra de Flaubert, Bouvard et Pécuchet, como una gigantesca reminiscencia o anamnesis: si no hay ciencia que permita aprender, entonces solo queda la fábula. Además, Platón (2000: 85c) llega a confirmar la existencia de esa forma de conocimiento basado en la reminiscencia tras una demostración realizada mediante un ejemplo de geometría, e implícitamente afirma: «El que no sabe, por lo tanto, acerca de las cosas que no sabe, ¿tiene opiniones verdaderas sobre eso que efectivamente no sabe?» (Platón, 2000: 307). Cierto que detrás de esta idea, mediada por la filosofía platónica, está la teoría de la inmortalidad y eternidad del alma, y la transmigración de la misma alma (2000: 85b-86b), pero puede tener su utilidad en el marco del transconocimiento narrativo.

En el caso de Flaubert se trata, en concreto, de la maraña en que quedan atrapados los protagonistas por propia iniciativa, del des-conocimiento que resulta de sus proyectos, y por tanto de la improbabilidad del razonamiento, o sea de la ciencia imposible. Pero no debe olvidarse que, al mismo tiempo, también hay una evidencia plausible y hasta excitante en relación con este epistemotexto: entonces la fábula factible es lo único posible, se demuestra la facticidad del relato titulado Bouvard et Pécuchet, y se hace posible el hecho de que un relato pueda convertirse en conocimiento en sí mismo y por sí mismo, y más concretamente todavía en trans-conocimiento narrativo.

En su libro titulado Conocimiento prohibido, Roger Shattuck ha venido a plantear una hipótesis altamente interesante en relación con el problema del conocimiento en connivencia con la literatura: el conocimiento que él denomina «prohibido». Si bien se mira, las desgraciadas aventuras de Bouvard y Pécuchet persiguiendo el conocimiento y su absoluto y estrepitoso fracaso encuentran en la teoría de Shattuck unas preguntas realmente inquietantes, aunque no por ello carentes de sentido. Para empezar, baste esta cuestión más bien general: «¿Es posible decidir si hay formas de conocimiento, verdadero o no verdadero, que por alguna razón no debiéramos conocer?» (Shattuck, 1998: 21). A la que podrían seguir algunas más: «¿Es la curiosidad el único impulso humano que no debe nunca restringirse? ¿O encarna la mayor amenaza a nuestra supervivencia como seres humanos?» (Shattuck, 1998: 22). De estas cuestiones podrían derivar otras preguntas más específicas, aunque no menos inquietantes en lo que se refiere a la 
interpretación de la obra en cuestión, protagonizada por Bouvard y Pécuchet: ¿Qué pasa entonces con la relación, ciertamente dicotómica, entre conocimiento y verdad? ¿A fin de cuentas, para qué sirve el conocimiento en este caso? ¿En realidad, tanto tiempo, dinero y esfuerzo derrochados para qué?

Pues bien, siempre que se tenga presente, por ejemplo, el avance del conocimiento que se ha producido en el proceso histórico, la dimensión que adquiere aquí la construcción por parte del autor de un mundo posible de la ficción y la distanciación narrativa en clave hermenéutica que permite comprender este epistemotexto, la única respuesta (aparte de la curiosidad), aunque bien paradójica, es que ese relato (científico) de un fracaso tiene un sentido (narrativo) en un relato de ficción literaria. Y, por cierto, una última pregunta de Shattuck deja la cuestión abierta, pero bien orientada hacia una semántica ficcional de amplio espectro (en la que entrará la segunda parte de este trabajo), que de algún modo se deja intuir en otra pregunta del autor: «¿Puede la imaginación por sí sola sostenernos realmente si falla la realidad?» (Shattuck, 1998: 169).

Dicho esto, resulta evidente la desproporción - paradójica- entre, por una parte, el entusiasmo inicial de los protagonistas Bouvard y Pécuchet: «Ensuite, ils glorifièrent les avantages des sciences: que de choses à connaître, que de recherches... si on avait le temps ! Hélas !» (Flaubert, 1966: 34); y por otra parte la ridiculez de sus proyectos, como lo demuestra bien a las claras este último, absolutamente decepcionante: «Alors Bouvard et Pécuchet voulurent se signaler par une œuvre qui, forçant les respects, étonnerait, éblouirait leurs concitoyens, et ils ne trouvèrent pas autre chose que des projets d'embellissement pour Chavignolles» (Flaubert, 1966: 324), sin olvidar los fracasos a que sistemáticamente se ven condenados todos los proyectos narrados en la obra.

Así pues, todo consiste en una vasta exposición, muy detallada, de múltiples útiles epistémicos disponibles en la época, sin que de la narración se derive malgré tout un avance para el ser humano: no debe olvidarse que ambos protagonistas son copistas, no son científicos, ni siquiera representan a unos seres reflexivos capaces de reproducir las dudas de la época sobre diversas cuestiones. En efecto, el libro recorre transversalmente multitud de técnicas y conocimientos, sin ofrecer una salida lógica, coherente, constructiva, y menos aún científica. De algún modo, entonces, la obra de Flaubert cuenta un relato epistemotextual basado en una desconstrucción del conocimiento, que solo produce al final desconocimiento y desolación en sus apesadumbrados protagonistas, y acaso también en el desconcertado lector.

En realidad, las aventuras de Bouvard y Pécuchet con los saberes y el conocimiento no acaban de encajar en ninguna de las categorías planteadas por Shattuck (1998: 391-402), ya que no se trata de ningún tipo de conocimiento «inacessible», «prohibido», «peligroso», «frágil», «doblemente prohibido» ni «ambiguo». Eso sí, Bouvard y Pécuchet tienen una relación conflictiva con el saber, pero no llegan ni por asomo a acceder al conocimiento, simplemente se quedan en un estadio previo al saber que solo se puede calificar, simple y llanamente, de «sottise humaine», tal como señala Jacques Suffel: «[Bouvard et Pécuchet] se 
promènent à travers la connaissance, entassant les erreurs et les découvertes, ne rencontrant partout qu'incertitude, ineptie ou méchanceté» (Flaubert, 1966: 1314).

De algún modo, tras los sucesivos y tormentosos fracasos, a estos atribulados personajes solo les queda la opción de copiar, es decir volver a su antiguo oficio, pero también realizar una acción que se opone frontalmente al saber, al conocimiento, a la investigación. En principio, copiar es lo contrario de investigar y de saber, la copia anula todo conocimiento, es la destrucción del principio básico del saber. Por eso el proyecto del relato de Bouvard et Pécuchet coincide con otro proyecto no menos importante, y disparatado, el Dictionnaire des idées reçues, una especie de diccionario de la «sottise».

Respecto a este último, y por extensión también a Bouvard et Pécuchet, en una carta a Louise Colet del 16 de diciembre de 1852, las opiniones de Flaubert no dejan lugar a duda:

Ce serait la glorification historique de tout ce qu'on approuve. J'y démontrerais que les majorités ont toujours eu raison, les minorités toujours tort. J'immolerais les grands hommes à tous les imbéciles [...] Cette apologie de la canaillerie humaine sur toutes ses faces, ironique et hurlante d'un bout à l'autre, pleine de citations, de preuves (qui prouveraient le contraire) et de textes effrayants (ce serait facile), est dans le but, dirais-je, d'en finir une fois pour toutes avec les excentricités, quelles qu'elles soient. [...] Je crois que l'ensemble serait formidable comme plomb. Il faudrait que, dans tout le cours du livre, il n'y eût pas un mot de mon cru (Flaubert, 1998: 213-214).

Como bien intuye Suffel, de este modo Flaubert se vengaría de sus contemporáneos, sobre todo de los burgueses, cuya incompetencia epistémica en distintas materias (agricultura, literatura, medicina, arqueología, historia, política) le tenía profundamente disgustado. Y de nuevo el absurdo proyecto de construir el conocimiento (científico) viene a fracasar, permitiendo de paso, paradójicamente, la validez del relato (literario), un epistemotexto, como transconocimiento narrativo. De este modo, Flaubert pone en cuestión el régimen de conocimiento y de verdad que ya se está desmoronando en aquellas fechas de finales del siglo XIX con la caída de la cultura enciclopedista, que bien podría definirse así:

Le procès d'une culture qui ne fait qu'accumuler et inventorier. Les tentations de l'encyclopédisme, la multiplication de « connaissances », l'ingurgitation omnivore de «faits »voilà le syndrome d'une maladie qui mène droit au relativisme paralysant, à la stérilité, au désespoir (Brombert, 1971: 172).

Como bien concluye Victor Brombert, a propósito de La tentation de saint Antoine, en un análisis igualmente aplicable a Bouvard et Pécuchet, se trata de una «libido sciendi menant au nihilisme»(Brombert, 1971: 120). Y de nuevo, paradójicamente, la literatura, a pesar de narrar una historia del fracaso de multi- 
tud de saberes y de la incapacidad para conocerlos, al narrar precisamente eso mismo, se constituye en una especie de meta-saber que se adelanta a los acontecimientos y a la historia real de la humanidad actual.

\section{Literatura «como» ciencia}

\subsection{El epistemotexto ficcional narrativo}

Como también ocurre con otros ámbitos de las ciencias humanas en relación con el conocimiento, la literatura es necesaria para entender la realidad, asaz compleja, en la que está inmersa la humanidad en cada momento histórico, y también para saber enfrentar los desafíos del futuro (transconocimiento ficcional). El mismo Jonah Lehrer, cuando describe la influencia ejercida por el pensamiento de Henri Bergson sobre Marcel Proust, señala que «la realidad se comprende mejor subjetivamente: a sus verdades se accede de manera intuitiva» (Lehrer, 2010: 106). Con lo cual viene a demostrar la capacidad de la literatura para interactuar primeramente con la filosofía y luego con la ciencia, pasando mientras tanto por todo el relato que descubre las verdades del interior humano. Algo parecido es lo que sostiene Tzvetan Todorov (2009: 28) cuando afirma lo siguiente: «El conocimiento de la literatura no es un fin en sí, sino una de las grandes vías que llevan a la realización personal».

En un sentido amplio, la literatura aporta ya de entrada un conocimiento profundo sobre lo humano y lo social, la experiencia particular y al mismo tiempo general sobre el mundo tanto en el nivel sensible como en el experiencial, multitud de datos sobre los comportamientos humanos, y también todo tipo de elucubraciones (hipóstasis) sobre fenómenos conocidos y desconocidos, y hasta cierta pre-visión e hipótesis sobre saberes no conocidos aún. Para Antoine Compagnon, la literatura es un conocimiento específico y totalmente diferenciado de la filosofía y de la ciencia: «Este conocimiento tiene por objeto lo que es general, probable o verosímil, la doxa, las sentencias y máximas que permiten comprender y regular el comportamiento humano y la vida social» (Compagnon, 2015: 37-38). Es más, este tipo de conocimiento precede a todos los demás conocimientos, lo cual hace suponer que sería la base de todos ellos, sin olvidar que, consiguientemente, para él la literatura tiene una función de aprendizaje cuyo objeto viene a definir del siguiente modo: «Hay un conocimiento del mundo y del ser humano que nos proporciona la experiencia literaria» (Compagnon, 2015: 38).

Por otra parte, no es menos cierto que la obra literaria -sobre todo la moderna- suele ser muy propensa a poner en evidencia su propio origen y funcionamiento, como si en esas fases autonímicas de la textualidad se encontrara la clave de un posible desarrollo epistémico de la obra. Un ejemplo paradigmático podría encontrarse implícitamente en la definición de «inter-texto» de Roland Barthes: para definir el inter-texto como recuerdo circular, evocación lectural y espontánea dentro de una cultura literaria, provocado por la presencia in mente de una obra de referencia perteneciente a la cosmogonía literaria del sujeto lector, Barthes (1973: 59) emplea curiosamente el término máthesis (acto de conocimiento), que permite al lector interpretante la asociación de obras diferentes gracias a su competencia 
lecto-cultural. La máthesis sería entonces lo que permite poner en relación los textos del inter-texto, y por el mismo efecto, propio de la literatura y la lectura, también sería lo que pone en relación de interacción la literatura y el conocimiento, los saberes; pero, en este caso, ese acto de conocimiento tendría lugar dentro de la propia literatura, o sea que la literatura sería ya conocimiento en sí misma y por sí misma.

Por lo tanto, no se podría dudar de que la literatura, en su relación multisecular con la filosofía primero, y con todo tipo de ciencias después, se halle en interacción con el conocimiento y esté dotada de una función epistémica: el impulso creativo que conduce a la construcción de los mundos posibles de la ficción en muchos casos se produce paralelamente a la representación de un saber acumulado en una tradición multisecular. La literatura, en su dimensión habitual y mayoritaria, relacionada con los mundos posibles de la ficción, suele implicar una visión del mundo por la cual se podría producir la transformación de ese mismo mundo. Así que el mundo posible de la ficción propuesto por la literatura va ligado a un aparato epistémico.

En este sentido, la hipótesis aquí presentada va más allá de la noción de «epistemología literaria», según la cual es posible desplegar un «conocimiento científico sobre la creación y crítica metacientífica sobre la ciencia de la literatura» (Bobes, 2008: 16): aunque la autora ciertamente abre la posibilidad de un despliegue epistémico de la obra literaria, se trata de un desarrollo específicamente metaliterario, y por lo tanto implícito, unidimensional. Más cercana resulta sin duda la noción de «escritura sapiencial» de Harold Bloom, según la cual ciertas obras literarias tienen «sus propios criterios implícitos de fuerza estética y cognitiva» (Bloom, 2005: 16, el resaltado es mío), o sea que son capaces de construir conocimiento por sí mismas.

La literatura también es capaz de generar la construcción de nuevos mundos por vía ficcional. Pero el mismo discurso que produce narraciones, produce también ideas, conceptos, definiciones, teorías o nociones, incluso dentro del propio discurso narrativo y en perfecta fusión con él. Así pues, la ficción, mediante la construcción u organización de mundos posibles, entrega al lector no solo esos mismos mundos imaginados como un relato, sino también todo el saber que llevan asociados para que, efectivamente, sean posibles. No en vano, entonces, los hermanos Goncourt, en su prefacio a Germinie Lacerteux (1864), anunciaban la llegada de una nueva novela con vocación científica:

Aujourd'hui que le Roman s'élargit et grandit, qu'il commence à être la grande forme sérieuse, passionnée, vivante, de l'étude littéraire et de l'enquête sociale, qu'il devient, par l'analyse et par la recherche psychologique, l'Histoire morale contemporaine, aujourd'hui que le Roman s'est imposé les études et les devoirs de la science, il peut en revendiquer les libertés et les franchises (Goncourt, 1990: 56, el resaltado es mío).

Desde la teoría de la ficcionalidad y, en concreto, desde la teoría de los mundos posibles de la ficción, ya se señala la pertinencia de esta hipótesis y la 
operatividad de la transferencia epistémica realizada desde la literatura hacia el mundo: «En una dirección, al construir mundos ficcionales, la imaginación poética trabaja con "materiales" extraídos de la realidad; en dirección contraria, las construcciones ficcionales influyen profundamente en nuestra representación y comprensión de la realidad» (Dolezel, 1999: 11).

Además, desde la teoría del conocimiento ya se apunta, en primer lugar, una distinción entre los dominios epistémicos de la filosofía y de la literatura, ya que sus métodos son bien diferentes: «El poeta y el artista no están atentos directamente a la totalidad del ser, como el filósofo. Su espíritu se dirige, en primer término, a un ser y un proceso concretos» (Hessen, 1981: 19). Pero es que, en segundo lugar, esos objetos concretos afectados por la operación epistémica pasan por un tratamiento fenomenológico especial que no coincide con la realidad sino con la irrealidad, o sea lo ficcional: «Y al representar éstos, los elevan a la esfera de la apariencia, de lo irreal» (Hessen, 1981: 19). En tercer lugar, la maravilla de la literatura es que, a pesar de construir sus mundos posibles en el ámbito de lo ficcional (irreal), lo que construye es recepcionado y aceptado como si fuera real, es decir que el conocimiento «ficcional» tiene una incidencia sobre el conocimiento «natural», propio de los saberes humanos: «Lo peculiar de esta representación consiste en que en este proceso irreal se manifiesta el sentido del proceso real; [...] interpretando primordialmente un ser o un proceso particulares, dan indirectamente una interpretación del conjunto del universo y de la vida» (Hessen, 1981: 19).

Llegados a este punto, cabe por tanto afirmar que la literatura puede constituir también, por sí misma y en sí misma, una vía factible de construcción del conocimiento, puede ser un sistema alternativo o diferente de saber, otro sabersaber (un saber que se-sabe). Por cierto que, para algunos críticos, el problema de la representación y/o generación del saber por medio del texto ficcional narrativo podría quedar planteado en la dicotomía ficción/saber, en la que ya de entrada se atiende a la posibilidad de un saber propio de la ficción: «L'objet d'une fiction stricto sensu est quelque chose dont l'existence dépend de l'existence de la fiction elle-même. [...] Une fiction produit un objet fictif, devenant en même temps une source de savoir sur cet objet fictif» (Klinkert, 2012: 1).

Esta hipótesis extensiva de la noción básica de la epistemocrítica no es una idea lanzada gratuitamente al espacio de la especulación, sino que ha tenido ya cierto recorrido. Michel Pierssens señala que no hay una respuesta definitiva a la pregunta sobre qué es el saber, y por ello plantea explorar otro tipo de preguntas, como por ejemplo: «Est-il d'autres manières de forger un savoir sur le savoir?» (Pierssens, 2009: 1). En el contexto del argumento desarrollado por Pierssens, su respuesta implicaría que la literatura puede constituir efectivamente «esa otra manera» de acceder al saber, dando pie así a la fundación de la epistemocrítica como una vía de interpretación sobre el lugar que la obra literaria reserva al saber, en qué consiste exactamente esa producción «literaria», cómo logra enriquecerse con su paso por la ficción y si puede aprender algo sobre sí misma. 
La hipótesis de Thomas Klinkert antes citada se sustenta en la teoría de la doble función -estética y epistémica- del texto literario (epistemocrítico), según la cual «la fiction littéraire peut donc s'approprier un savoir qui existe en dehors d'elle-même, en employant une forme spécifique de codage» (Klinkert, 2012: 2). Para ello se basa en el principio de la «diferencia directriz» (Leitdifferenz) de Niklas Luhmann, aunque este punto de partida es modificado por una función de recodificación que permite efectivamente trans-ducir los elementos epistémicos, tras lo cual el texto literario podría representar y/o generar el saber. Todo ello guarda la debida coherencia con la literariedad, ya que en la ficción literaria «les lois de la communication sont partiellement suspendues» (Klinkert, 2012: 2), de modo que la ficción sería capaz entonces «d'imiter et de simuler tous les discours possibles qu'elle transforme en éléments formels» (Klinkert, 2012: 2). Y como el proceso que aquí tiene lugar resulta ser de «segundo grado» (es un saber sobre el saber), entonces el saber producido por medio de estas operaciones epistemoestéticas es un meta-saber: el trans-conocimiento narrativo (reflexivo, estético, ficcional) se hace así meta-conocimiento.

En una valoración de conjunto de las novelas de Michel Butor, Françoise Van Rossum-Guyon avanza unos elementos de epistemocrítica que, no por adelantados, dejan de tener su sentido preciso para el argumento que aquí pretendo desarrollar. Ciertamente la novela butoriana mantiene, en el nivel del contenido y las estructuras narrativas, ciertas relaciones con las matemáticas, el urbanismo, la arquitectura, el sistema educativo, la historia, el transporte ferroviario, etc., ya que ello era necesario para impulsar el nivel de formalización que Butor imprime a su escritura y constituye una de sus características más destacadas. Como bien se sabe, este intenso trabajo sobre la forma novelesca es un leitmotiv del autor y tiene su fundamento en una cuestión fenomenológica según la cual básicamente el escritor interactúa con el mundo a través de su obra: «L'importance du travail sur la forme dans le roman se fondait sur la constatation d'un décalage entre les formes de récit existantes et une réalité en perpétuelle transformation» (RossumGuyon, 1971: 163). Pero, dicho esto, todo ese trabajo de formalización que afecta a las estructuras narrativas de la novela butoriana, afecta también a la visión del mundo, porque «le roman [...] a pour effet de transformer la représentation que nous nous faisons du réel» (Rossum-Guyon, 1971: 170): por un lado la realidad provoca o impulsa la representación de la ficción, y por otro la novela reenvía de nuevo su mundo construido a la realidad en que el lector se sitúa mientras se representa todo lo narrado.

Si la realidad constituye el punto de partida del saber relatado por la literatura en sus epistemotextos al uso, inversamente, tal como señalaba Dolezel (1999: 11), se podría hablar de un saber narrativo o una estructura narrativa del saber surgida de un doble impulso fenomenológico y epistemológico a partir del cual el mundo posible de la ficción incide sobre el mundo real. Curiosamente, en relación con esta misma idea, desde el punto de vista de una teoría de la irrealidad literaria se puede argumentar lo siguiente: 
La única razón de ser de la literatura consiste en decir aquello que tan sólo la literatura puede decir. Se trata de esclarecer narrativamente el mundo de la vida aventurándose en el reino de las posibilidades humanas. El mundo real es iluminado cuando se le proyecta sobre la ficción de la posibilidad (Innerarity, 1995: 165).

De este modo, las estructuras mismas de la novela no son solamente una representación ficcional de lo real o un proyecto de re-construcción de la realidad en el nivel de la ficción, sino que se convierten, por arte de los mundos posibles de la ficción, en un modelo que revierte sus estructuras sobre lo real para de algún modo intervenir en el proceso de transformación de la realidad que el ser humano lleva a cabo sin cesar. Por tanto, aquí, la literatura no solo asimila interactivamente otros saberes, sino que en su laboratorio de escritura y relatos construye un saber propio (en el caso concreto de la novelística de Butor: estructural, organizativo, humano, social, ideológico, político) que trata de reenviar a la realidad para su propio beneficio y natural transformación.

Vistas las características del funcionamiento del transconocimiento en el epistemotexto literario, y tal como se ha de-mostrado a lo largo de este estudio, se podría abrir la posibilidad de adscribir la epistemocrítica a una «teoría del conocimiento especial» (según la clasificación de Hessen) ya que, desde una perspectiva general y traduciendo la definición en clave literaria, la epistemotextualidad «hace tema de investigaciones críticas los principios y conceptos fundamentales en que se expresa la referencia de nuestro pensamiento a los objetos» (Hessen, 1981: 21). De acuerdo con esta definición, la epistemocrítica pondría de relieve el hecho de que la literatura y la ciencia comparten al menos el objetivo de una «teoría de los principios materiales del conocimiento humano» (Hessen, 1981: 20). Desde un enfoque fenomenológico estricto, Hessen analiza el funcionamiento que hace posible la aprehensión del objeto por el sujeto que, aplicada a la referenciación o generación de un conocimiento por medio de la escritura-lectura, permite llegar a una conclusión de alto interés en relación con el epistemotexto literario. Dice Hessen que, al igual que los objetos reales o experienciales, «también estos objetos ideales poseen un ser en sí o trascendencia, en sentido epistemológico» (Hessen, 1981: 27, el resaltado es mío). O sea que los objetos imaginados por la literatura («ideales») también poseen una dimensión existencial e incluso pueden llegar a funcionar como los demás objetos del mundo: el epistemotexto literario constituye entonces un objeto (ficcional) que puede ser aprehendido igualmente por el sujeto cognoscente debido a que se comporta fenomenológica y epistémicamente igual que los demás objetos, dispone de una capacidad de existir equiparable -ontológicamente- a todos los objetos existentes, y por ello, a pesar de ser una construcción imaginaria, tiene una virtualidad existencial parecida a la de cualquier otra entidad.

Además, desde un punto de vista más amplio si cabe, subyace en esta problemática la noción de verdad, que es un concepto muy importante cuando se habla de conocimiento: no se olvide que, tras haber relacionado, más arriba, literatu- 
ra y ciencia en cuanto al proceso del conocimiento, Hessen se refiere a esa teoría como una «teoría del pensamiento verdadero» (Hessen, 1981: 21). Y ciertamente, más allá o más acá de las realidades exploradas y definidas por la ciencia, como si fuera una especie de metaciencia paralela o alternativa de la filosofía, la literatura explora y relata toda una serie de realidades como si fueran «verdades». Como bien señala Aldous Huxley: «El poeta puede expresar, puede evocar, puede incluso crear posibilidades de experiencia hasta entonces desconocidas o tal vez inexistentes» (Huxley, 2017: 51). La verdad, en tanto que conocimiento último, es perseguida sistemáticamente en toda obra literaria, es la verdad intrínseca de esa obra. Poco importa si es objetiva o subjetiva, según el caso, porque lo que importa es acceder a ese territorio todavía desconocido al que el ser humano no había podido llegar. Conviene recordar que el ser humano jamás agota su ansia de saber y descubrir nuevas realidades, y que el lenguaje, medio específico de la literatura, es un método explorador de infinitas posibilidades.

En relación con este problema, no se puede pasar por alto la referencia a la interdisciplinariedad como la dimensión apropiada para la adecuada interrelación o interacción de los distintos saberes:

La capacidad de actuar sobre el mundo es hoy una necesidad: todos somos testigos -y la mayoría víctimas- de que el mundo, la realidad social, la política y, sobre todo, la económica y la medioambiental, se nos escapan de las manos [...] la interdisciplinariedad no es un ejercicio académico, es un deber moral (Sánchez, 2011: 20-21).

Y el transconocimiento literario, tal como ha sido definido aquí, el objeto propio de la epistemocrítica, es el modo de realizar la adecuada transferencia interdisciplinar, «literatura " $y$ " ciencia»- de un saber a otro en el texto literario. Si además tenemos en cuenta la dimensión de la «literatura "como" ciencia», resulta que se abre un nuevo paradigma del conocimiento con la posibilidad de explorar todos los saberes que a la literatura se le antojen: «Si ciencia y literatura, si ciencia y lenguaje se hermanan, la causa de la interdisciplinariedad se verá favorecida» (Sánchez, 2011: 296), ampliando así o potenciando de paso esa misma interdisciplinariedad con nuevas opciones de transferencia sapiencial.

\subsection{Los relatos del conocimiento: la epistemoficción}

Desde el punto de vista de la epistemoficción, las estructuras epistemotextuales del transconocimiento autónomo de la obra literaria (generadas en su interior) son puestas a disposición del lector para que las transfiera al conocimiento general y puedan ser aplicadas al mundo real. A este respecto, como bien señala Yuri M. Lotman (1982: 270-271) desde la perspectiva más amplia de una semiótica de la cultura, pero perfectamente aplicable en este caso, «la estructura del espacio del texto se convierte en el modelo de la estructura del universo, y la sintagmática de los elementos en el interior del texto en el lenguaje de modelización espacial». Así que, mediante la transferencia del saber propio de la obra a la realidad mundana del lector, se opera un proceso en el que las maniobras de la semiosis en el interior del texto (semiótica) se transfieren a la mimesis o re-construcción 
de la realidad (semántica ficcional). En este mismo sentido, Georges Gusdorf (1993: 356) viene a aportar una interpretación similar por vía de la hermenéutica filosófica: «Depuis l'avènement de la conscience moderne avec Francis Bacon, la science consiste, pour un sujet doué de raison, à décrire la configuration de l'ordre des objets qu'il a sous les yeux».

En La vie mode d'emploi de Georges Perec el sistema referencialista vinculado al complejo de constricciones lógico-matemáticas, de inspiración oulipiana, aporta un bagaje de referencias epistémicas deslumbrante, abrumador. Así que, en consonancia, el narrador resulta ser un tanto objetivo, incluso distante, neutro, y muy detallista: relata pequeñas historias y no hay apenas movimiento, debido sobre todo a las abundantes y amplias descripciones y enumeraciones. Este narrador descriptivista se limita a representar escenas en las que los personajes permanecen quietos casi siempre (instantaneidad), como si la acción narrativa se hubiera reducido a una secuencia de fotografías. Y su tarea consiste sobre todo en describir el ambiente, la situación y todo el decorado donde se acumulan cientos y cientos de objetos. No hay un argumento de principio a fin, ni trama por tanto, solo breves historias a veces asociadas a objetos o a ciertos personajes; salvo la historia del proyecto de Bartlebooth, que se encuentra diseminada por todo el libro y es una metáfora, un tanto controvertida, del mismo libro. No hay un protagonista y mucho menos algo que se aproxime a un héroe, aunque llama la atención la nómina extensísima de personajes, la mayoría de ellos variopintos.

El narrador descriptivista aporta entonces una enorme cantidad de datos y detalles de multitud de objetos, fenómenos, experiencias e historias, en una gigantesca lista cuyos límites sería muy difícil fijar, y toca todos los temas posibles, dando lugar a una exposición realista (museo, catálogo) de todo lo que se acumula en el universo cotidiano de la humanidad en París en los años 1970. El afán taxonomista recuerda a Bouvard et Pécuchet, y continúa en cierto modo el esquema narrativo de la primera novela de Perec, Les Choses (1965). Y, sin que sea un juicio de valor a priori, todavía en 1978 el libro no puede evitar denotar en cierto modo la influencia del Nouveau Roman en cuanto a la estrategia objetal y sociológica que constituye su composición y se desprende en el acto de su recepción. En efecto, la taxonomía inunda decenas de páginas con listas casi interminables de productos de consumo. Así que el lector se enfrenta a una jungla inmensa de objetos de decoración que invaden los distintos espacios del edificio parisiense, convertido a la sazón en un auténtico museo de referencia de todo aquello que en los años 1970 pudiera constituir el hábitat íntimo más apropiado para la vida en París.

Sin duda hay en La vie mode d'emploi una idea metódica para describir con exactitud el hábitat parisiense de la época, de modo que así se responde a la pregunta: «¿cuáles son las instrucciones de uso para la vida?» Incluso se podría añadir que la práctica de la intertextualidad, en este caso proliferante, participa de este sistema narrativo descriptivista: las citas, referencias y alusiones reproducen textos como si fueran efectivamente otros tantos objetos que satisfacen el afán taxonómico del narrador descriptivista. No se olvide que la escritura es el crisol de 
donde surge algo nuevo que la trasciende, y este movimiento puede llevar implícito un impulso epistémico:

La démarche épistémocritique, ne se ramène donc pas à simplement repérer l'empreinte univoque et exclusive de telle ou telle "science" ou doctrine identifiable [...]. L'écriture est au contraire perçue ici à son tour comme le ferment d'une "crise" permanente des savoirs qu'elle mobilise-souvent à son insu (Pierssens, 1990: 13, el resaltado es mío).

Así pues, desde la perspectiva epistemocrítica, La vie mode d'emploi puede ser considerada, en sí misma, una obra basada en el proyecto epistemoficcional de conocer/descubrir el «modo de empleo» de la vida humana en París en el siglo XX. Dicho proyecto se lleva a cabo desde múltiples puntos de vista, proporcionados por la variedad inmensa de historias narradas y los saberes aportados en esas narraciones-descripciones (epistemoficción).

Desde un punto de vista más general, para Jean Starobinski se trataría una vez más de la «relación» por medio del lenguaje entre una conciencia singular y el mundo, por la cual la obra exporta su construcción ficcional al exterior de la literatura, al mundo en general, donde su aportación epistémica podrá ser recibida, acogida, rentabilizada. Este proceso en mi opinión se llevaría a cabo en tres fases. En primer lugar, aparece la posibilidad de una inferencia o hipóstasis creativa (heuresis, función epistémica) que permitiría la construcción de algo: «Es posible que la obra, mundo dentro de un mundo, se me presente como la expresión microcósmica del universo en que su nacimiento se produjo» (Starobinski, 1974: 16). En segundo lugar, se produce una asimilación o paralelismo constructor de la obra literaria en relación con el mundo, pues la obra construye un mundo a su modo, que es exportado fuera de la obra (transducción semiótica inversa del saber), se asimila al mundo exterior, donde se produce el encuentro del mundo de la obra (ficcional) y el mundo de lo que se llama en general mundo (real): «De forma que las relaciones diferenciadas en el interior de la obra se repiten fielmente fuera de la misma, en un mundo expandido del que ella es ya sólo un elemento» (Starobinski, 1974: 16). Y en tercer lugar, la obra adquiere una dimensión ontológica y fenomenológica, por la cual se configura como un mundo virtual y ficcional, pero existente y funcional, culminación de la epistemoficción en una semántica ficcional: «Cabría entonces la convicción de que la ley interior de la obra me ha proporcionado una síntesis simbólica de la ley colectiva del momento y del medio cultural en cuyo seno se produjo» (Starobinski, 1974: 16).

Así pues, en el contexto de esta narración descriptivista de La vie mode d'emploi, se introducen en el texto innumerables referencias epistémicas de un transconocimiento singular que surge directamente de esas «instrucciones de uso para la vida». Lugar destacado ocupa sin duda el mundo del arte, sobre todo la pintura, la gran pasión de Perec, a la que rendirá un emotivo homenaje, por ejemplo, justo al final del epílogo que cierra totalmente la novela de novelas que es $L a$ vie mode d'emploi, con la representación de la muerte del maestro-pintor de acua- 
relas que fue Valène, incluida la metáfora que encierra el sentido global de la novela y, para Perec, de toda obra de arte:

Une grande toile carrée de plus de deux mètres de côté était posée à côté de la fenêtre, réduisant de moitié l'espace étroit de la chambre de bonne où il avait passé la plus grande partie de sa vie. La toile était pratiquement vierge: quelques traits au fusain, soigneusement tracés, la divisaient en carrés réguliers, esquisse d'un plan en coupe d'un immeuble qu'aucune figure, désormais, ne viendrait habiter (Perec, 1982: 602).

Aunque, sin perjuicio de lo anterior y siguiendo una tradición secular en literatura, no ocupa un lugar menos importante la referencia a la medicina (endocrinología, toxicología, anatomía, traumatología, farmacología, oftalmología). Por su parte, la arquitectura y el urbanismo se hallan también referenciados, ya que $L a$ vie mode d'emploi se inscribe en una travesía temática literaria de cierta importancia -los edificios-novelas- junto a Le père Goriot de Honoré de Balzac, Potbouille de Émile Zola o Passage de Milan de Michel Butor por ejemplo. El sistema de referenciación epistémica se extiende tanto como la amplitud de la descripción y alcanza a la ingeniería, las matemáticas, la arqueología, la religión, la gastronomía, la zoología, la bibliografía, la etnografía, la historia, el marketing, la lingüística o la economía.

Ahora bien, no se puede pasar por alto, en fin, la referencia a otra dimensión de la epistemoficción como es el transconocimiento «metatextual» conformado por la presencia en el libro de alusiones al juego del Go, el puzzle o la Gestalttheorie. Esta aportación no solo constituye un conjunto de referentes epistémicos del transconocimiento junto a los ya citados más arriba, sino un dispositivo metacientífico por el cual la obra misma se convierte en generadora de un saber sobre sí misma, su génesis y proceso de construcción, y en relación también con un nuevo mundo posible de la ficción que habrá de incidir, luego, en el mundo real. Por lo tanto, junto al aparato epistémico del transconocimiento epistemotextual constituido por toda la red de referencias del sistema descriptivista impuesto a machamartillo por el peculiar narrador de La vie mode d'emploi, la parte epistemotextual de esos referentes metatextuales de la obra también se incorporan al epistemotexto total, aunque modificando su extensión y alcance, pues a través de ellos se accede a un cierto nivel de autonomía del conocimiento generado por la misma obra desde su propia autognosis (epistemoficción).

Como bien señala Pierssens, «la littérature pense et agit» (Pierssens, 1990: 14), la obra literaria es al mismo tiempo un lugar reflexivo, imaginativo, y un lugar heurístico, creativo, constructor. Por su parte, en su ensayo ya citado, Jean Starobinski no puede dejar de subrayar que la propia obra literaria tiene una dimensión epistemológica, pues así lo demuestra el hecho de que el primer acto de conocimiento realizado en la obra concierne a la obra misma (metatextualidad). Este acto reflejo de la obra literaria -y en esto La vie mode d'emploi resulta ser paradigmática- constituye su especificidad metaliteraria (la obra dentro de la obra, el saber sobre sí misma), pero sobre todo pone en evidencia el acto de cono- 
cer que supone toda escritura y toda lectura, y el mecanismo por el cual el texto es ciertamente un crisol de conocimiento: «No existe ninguna obra moderna que no lleve en sí el índice o la justificación de su propia venida al mundo [...]. Es preciso descifrar en la obra la naturaleza específica de un deseo, de un poder (de un genio), que ha tratado de alcanzarse a sí mismo y testimoniarse dando vida a la obra» (Starobinski, 1974: 20).

De hecho, como ya he avanzado más arriba, el sistema conjuntado de las constricciones oulipianas que sostiene la estructura narrativa de La vie mode d'emploi también se constituye, de entrada, en un meta-conocimiento a partir del cual se transfiere un saber de origen y adscripción «literaria» al conjunto del saber operativo en la realidad, con el ánimo de transformarla epistemoficcionalmente. Así pues, este transconocimiento de origen metatextual está basado en una serie de dispositivos metatextuales lógico-matemáticos, que a continuación paso a describir someramente:

a) El juego del puzzle, metáfora de las 99 piezas o capítulos del libro: una pieza es un capítulo, y cada capítulo tiene una «forma» (función, composición) que encaja con los demás para formar un todo -Gestalttheorie- que es la obra en su conjunto.

b) Un referente arquitectónico y urbanístico concretado en un edificio (ficcional) situado en la calle Simon Crubellier $\mathrm{n}^{\circ} 11$, una metáfora arquitectónica que se superpone con la novela misma, ya que los espacios arquitectónicos coinciden con los capítulos de la novela.

c) La fórmula matemática (combinatoria) del bicuadrado latino ortogonal de orden 10, basado en 10x10 cifras que no se repiten y van ordenadas en secuencias completas, es una ecuación cuya factibilidad había sido refutada por Leonard Euler, y que los matemáticos R.C. Bose, E.T. Parker y S.S. Shrikhande descubrieron en 1960; en la novela de Perec, este bicuadrado regula la combinación de cifras correspondientes a las listas de objetos, con la cual el autor elaboró el guión de cada capítulo dentro de la estructura narrativa.

d) Otra fórmula matemática, adaptada de la permutación, es la pseudoquenina de orden 10: un procedimiento combinatorio, por permutación secuencial, que permite corregir las repeticiones provocadas por el bicuadrado y, de este modo, la cantidad enorme de objetos que se incorpora a la narración sin repetirse.

e) La poligrafía del caballo: movimiento en forma de L de la pieza del caballo en el juego del ajedrez, regula el itinerario narrativo del edificio-novela (estructura narrativa), ordenando la equivalencia superpuesta del relato y del edificiopuzzle, como así lo demuestra el final de la propia novela:

C'est le vingt-trois juin mille neuf cent soixante-quinze et il va être huit heures du soir. Assis devant son puzzle, Bartlebooth vient de mourir. Sur le drap de la table, quelque part dans le ciel crépusculaire du quatre cent trente-neuvième puzzle, le trou noir de la seule pièce non encore posée dessine la silhouette presque parfaite d'un X. Mais la pièce que le mort tient entre ses doigts a la forme, depuis longtemps prévisible dans son ironie même, d'un W (Perec, 1982: 600). 
Ciertamente se podría definir La vie mode d'emploi como una obra en proceso de composición, una estructura narrativa compleja fundada en un mecanismo cuya operatividad se extiende desde su escritura hasta su lectura, en la que el receptor se verá invitado a operar por medio de los mecanismos disponibles en la propia ficción. Se abre así la vía de una «epistemosemiótica» dentro de la epistemocrítica, y en ella se trataría de analizar los signos del conocimiento en la obra de arte (epistemosemiosis). La vie mode d'emploi es, entonces, una epistemoficción construida sobre la base -abierta, desplegable- de una obra en movimiento capaz de poner en juego de permutación y combinatoria de una ingente cantidad de componentes. Hablamos entonces de un principio de construcción que no es estático, sino dinámico, tal como Yuri Tynianov anunciaba en su ensayo seminal: «L'unité de l'œuvre n'est pas une entité symétrique et close, mais une intégrité dynamique ayant son propre déroulement; ses éléments ne sont pas liés par un signe d'égalité et d'addition, mais par un signe dynamique de corrélation et d'intégration» (Tynianov, 1965: 117).

En su célebre ensayo Opera aperta, Umberto Eco habla de «obras abiertas» en el sentido de obras «no acabadas» que el autor entrega al intérprete lector al modo de un programa productivo. No se trata simplemente de la posibilidad de interpretar libremente una obra, algo que ocurre efectivamente en cualquier caso, sino que se ha dejado la obra en una cierta indefinición o ambigüedad para que el intérprete termine la tarea de una construcción siempre en marcha, que afecta incluso a su organización y estructura, a la manera de una colaboración: «Las actuales obras en movimiento tratan, en cambio, de instaurar armónicas y concretas relaciones de convivencia y entrenamientos de la sensibilidad y la imaginación, sin pretender constituir sustitutos órficos del conocimiento» (Eco, 1985: 78). Efectivamente, Eco descubre en las obras abiertas una posibilidad de conocimiento, pero en una primera fase lo relativiza frente al saber científico: «Es siempre arriesgado sostener que la metáfora o el símbolo poético, la realidad sonora o la forma plástica, constituyen instumentos de conocimiento de lo real más profundos que los recursos prestados por la lógica. El conocimiento del mundo tiene en la ciencia su canal autorizado» (Eco, 1985: 78). Esta relativización del saber artístico surgido de la apertura de la obra le lleva por consiguiente a una conclusión como esta: «El arte, más que "conocer" el mundo, "produce" complementos del mundo, formas autónomas que se añaden a las existentes exhibiendo leyes propias y vida personal» (Eco, 1985: 78). Pero a renglón seguido apunta a algo más importante que el simple sustituto del conocimiento científico de la obra, al referirse a la «metáfora epistemológica», es decir que «en cada siglo, el modo de estructurar las formas del arte refleja - a guisa de semejanza, de metaforización, de apunte de resolución del concepto en figura- el modo como la ciencia o, sin más, la cultura de la época ven la realidad» (Eco, 1985: 79). Es más, Eco, siguiendo el sendero abierto por medio del concepto de metáfora epistemológica, llegará a plantear una hipótesis avanzada del conocimiento de las obras artísticas, según la cual en esas obras abiertas o en movimiento, en cuyo goce o interpretación nunca se repite la 
misma producción o resultado, se encontrarán «las resonancias vagas o precisas de algunas tendencias de la ciencia contemporánea» (Eco, 1985: 80). De hecho, no vacilará en aludir a algunas características compartidas del conocimiento en esas obras y en la ciencia contemporánea: la indeterminación, la discontinuidad, la complementariedad, la modelización, la incompletud, la ambigüedad, la simultaneidad, la potencialidad, la posibilidad, la movilidad, la informatividad, la permutabilidad o la informalidad.

En esta consideración de la obra como metáfora epistemológica, Eco apela entonces a «resoluciones estructurales de una difusa conciencia teórica» (Eco, 1985: 176), pero que no suponen una apuesta menor por el conocimiento, ya que «representan la repercusión, en la actividad formativa, de determinadas adquisiciones de las metodologías científicas contemporáneas, la confirmación, en el arte, de las categorías de indeterminación, de distribución estadística, que regulan la interpretación de los hechos naturales» (Eco, 1985: 176). De este modo, Eco admite la validez de las obras abiertas para impulsar un cierto nivel de conocimiento que, en el caso de La vida instrucciones de uso, bien se podría definir como «la metaforización estructural de cierta visión de las cosas» (Eco, 1985: 177).

Además, el conjunto de relatos de La vie mode d'emploi remite al hecho de que la ficción se autoexamina en su propio funcionamiento (metaficción), pero enseguida se descubre que este conocimiento surgido del interior de la misma narratividad remite a un conocimiento que tiene que ver con la construcción de un mundo posible (ficcionalidad), en este caso relacionado con el saber de la vida humana (antropología literaria), un principio fundamental del humanismo. En efecto, este conocimiento en principio no proviene de otro ámbito o campo epistémico ajeno a la literatura para ser referenciado en el texto, sino que constituye en sí mismo un conocimiento propio enraizado en dos operaciones constructoras típicas de la literatura y base de la epistemoficción en este caso: la textualidad y la narratividad de La vie mode d'emploi. Si la literatura construye efectivamente un mundo posible es porque ahí reside un conocimiento que ha hecho posible precisamente esa creación: "La littérature [est] l'un des instruments essentiels qui permettent de penser ce monde: si elle ne le reflète pas, elle le "réfléchit". En ce sens, la littérature ne devrait pouvoir se comparer qu'à la science ou à la philosophie» (Pierssens, 1990: 165, el resaltado es mío).

En síntesis, la ficcionalidad se constituye así en un campo epistémico propio (epistemoficción) que no se limita a instrumentalizar otros saberes (tékne) para avanzar en su conocimiento, sino que construye o genera su propio ámbito epistemoficcional para hacer posible su propio conocimiento (episteme). Este nuevo campo epistémico propio de lo literario encuentra sus raíces en la capacidad de autognosis de toda obra artística, en la que el autor emprende el descubrimiento de sí mismo y de su relación con el mundo, pero también supone un proceso de construcción de experiencias y saberes que se asimilan a su vez a los mundos posibles de la ficción. Así se instituye un nuevo régimen de lo real que proviene de lo ficcional, antes considerado irreal paradójicamente. 
Y es que la clave de este proceso «reflexivo»y no tan solo «reflejo», la autognosis, entendida como motor de la construcción de conocimiento, tiene relación con la autopoiesis, un concepto procedente de la biología, pero que explica meridianamente cómo se producen los procesos constructores de los seres humanos en el caso que nos ocupa: «Todo lo que ocurre en los seres humanos, cuando se comportan como unidades discretas que generan operaciones [...] sólo hace referencia a ellos mismos [...] como si fueran entidades auto-referidas» (Maturana y Varela, 2004: 12). Entonces, más allá o más acá -según se mire- de todo el entramado de saberes que rodea y hace posible el conocimiento en el proceso de construcción de una obra literaria (enfoque epistemocrítico), se encuentra el núcleo generador de un conocimiento propio de esa obra, sea en relación con su mecanismo de construcción y su funcionamiento interno (metaficción), sea en relación con un saber innovado que es aportado en ese proceso como consecuencia de un impulso heurístico. Este saber, nacido en el seno mismo de la obra y no tan solo o no ya de una influencia externa, define pues un campo epistémico autónomo (epistemoficción), diferenciado respecto de los mecanismos epistemotextuales habituales.

\section{REFERENCIAS BIBLIOGRÁFICAS}

BARTHES, Roland (1973): Le plaisir du texte. París, Seuil.

BoBes, Ma Carmen (2008): Crítica del conocimiento literario. Madrid, Arco.

BLOOM, Harold (2005 [2004]): ¿Dónde se encuentra la sabiduría? Traducción de Damián Alou. Madrid, Taurus.

BROMBERT, Victor (1971): Flaubert par lui-même. París, Seuil.

CAmARERO, Jesús (2015): Epistemocrítica. Sarrebruck, EAE-OmniScriptum GmbH \& Co. KG.

Compagnon, Antoine (2015 [1998]): El demonio de la teoría. Traducción de Manuel Arranz. Barcelona, Acantilado.

CURTIUS, Ernst R. (1981 [1948]): Literatura europea y Edad Media latina. Traducción de Margit Frenk \& Antonio Alatorre. Madrid, FCE.

DOLEZEL, Lubomir (1999 [1998]): Heterocósmica. Ficción y mundos posibles. Traducción de Félix Rodríguez. Madrid, Arco.

ECO, Umberto (1985 [1962]): Obra abierta. Traducción de Roser Berdagué. Barcelona, RBA.

FlauberT, Gustave (1966 [1881]): Bouvard et Pécuchet. París, Garnier-Flammarion.

FLAUBERT, Gustave (1998): Correspondance. París, Gallimard.

GONCOURT, Edmond \& Jules de (1990 [1864]): Germinie Lacerteux. París, Flammarion.

GUSDORF, Georges (1993 [1982]): Le Romantisme I. París, Payot.

HeSSEN, Johannes (1981 [1925]): Teoría del conocimiento. Traducción de José Gaos. Madrid, Espasa-Calpe. 
HuXLEY, Aldous (2017 [1963]): Literatura y ciencia. Traducción de Roberto Ramos. Barcelona, Página indómita.

INNERARITY, Daniel (1995): La irrealidad literaria. Pamplona, Eunsa.

KLINKERT, Thomas (2012): «Fiction et savoir. La dimension épistémologique du texte littéraire au $\mathrm{XX}^{\mathrm{e}}$ siècle: Marcel Proust». Épistémocritique, 10. URL: http://epistemocritique.org/fiction-et-savoir.

KOYRÉ, Alexandre (1962 [1957]): Du monde clos à l'univers infini. París, Gallimard.

KuHN, Thomas S. (1995 [1962]): La estructura de las revoluciones científicas. Traducción de Carlos Solís. Madrid, FCE.

LeHRER, Jonah (2010 [2007]): Proust y la neurociencia. Traducción de Bernardo Moreno. Madrid, Paidós.

LotMan, Yuri M. (1982 [1970]): La estructura del texto artístico. Traducción de Victoriano Imbert. Madrid, Itsmo.

LYOTARD, Jean-François (1984 [1979]): La condición postmoderna. Informe sobre el saber. Traducción de Mariano Antolín. Madrid, Cátedra.

Maturana, Humberto \& Francisco VAREla (2004): De máquinas y seres vivos. Autopoiesis: la organización de lo vivo. Buenos Aires, Lumen.

PEREC, Georges (1982 [1978]): La vie mode d'emploi. París, Poche.

PIERSSENS, Michel (1990): Savoirs à l'œuvre. Essais d'épistémocritique. Lille, PU.

PIERSSENS, Michel (2009): «Proust au laboratoire». Épistémocritique, 4. URL: http://epistemocritique.org/proust-au-laboratoire.

Platón (2000 [386 ane]): Menón. Traducción de F. J. Oliveri. Madrid, Gredos.

REMAK, Henry H.H. (1998 [1961]): «La literatura comparada: definición y función», in $\mathrm{M}^{\mathrm{a}}$ José Vega, Neus Carbonell, La literatura comparada: principios y métodos. Traducción de $\mathrm{M}^{\mathrm{a}}$ José Vega. Madrid, Gredos, 89-99.

ROSSUM-GUYON, Françoise van (1971): «Michel Butor. Le roman comme instrument de connaissance», in Michel Mansuy (prés.), Positions et oppositions sur le roman contemporain. París, Klincksieck, 163-180.

SÁNCHEZ, José M. (2011): La nueva Ilustración: ciencia, tecnología y humanidades en un mundo interdisciplinar. Oviedo, Nóbel.

Shattuck, Roger (1998): Conocimiento prohibido. Traducción de Eva Rodríguez. Madrid, Taurus.

STAROBINSKI, Jean (1974 [1970]): La relación crítica. Traducción de Carlos Rodríguez. Madrid, Taurus.

TODOROV, Tzvetan (2009 [2007]): La literatura en peligro. Traducción de Noemí Sobregués. Barcelona, Círculo de lectores.

TYNIANOV, Yuri (1965 [1923]): «La notion de construction», in Tzvetan Todorov (comp.), Théorie de la littérature, París, Seuil,114-119.

VERNE, Jules (2002 [1879]): Les cinq cents millions de la Bégum. París, Poche. 